\title{
Masker phase effects in normal-hearing and hearing-impaired listeners: evidence for peripheral compression at low signal frequencies
}

\author{
Oxenham, Andrew J.; Dau, Torsten
}

Published in:

Journal of the Acoustical Society of America

Link to article, DOI:

$10.1121 / 1.1786852$

Publication date:

2004

Document Version

Publisher's PDF, also known as Version of record

Link back to DTU Orbit

Citation (APA):

Oxenham, A. J., \& Dau, T. (2004). Masker phase effects in normal-hearing and hearing-impaired listeners: evidence for peripheral compression at low signal frequencies. Journal of the Acoustical Society of America, 116(4), 2248-2257. https://doi.org/10.1121/1.1786852

\section{General rights}

Copyright and moral rights for the publications made accessible in the public portal are retained by the authors and/or other copyright owners and it is a condition of accessing publications that users recognise and abide by the legal requirements associated with these rights.

- Users may download and print one copy of any publication from the public portal for the purpose of private study or research.

- You may not further distribute the material or use it for any profit-making activity or commercial gain

- You may freely distribute the URL identifying the publication in the public portal 


\title{
Masker phase effects in normal-hearing and hearing-impaired listeners: Evidence for peripheral compression at low signal frequencies
}

\author{
Andrew J. Oxenham ${ }^{\text {a) }}$ \\ Research Laboratory of Electronics, and Harvard-MIT Division of Health Sciences and Technology, \\ Speech and Hearing Bioscience and Technology Program, Massachusetts Institute of Technology, \\ Cambridge, Massachusetts 02139 \\ Torsten Dau \\ Centre for Applied Hearing Research, Acoustic Technology, Ørsted•DTU, Technical University of Denmark, \\ DK-2800 Lyngby, Denmark
}

(Received 19 December 2003; revised 29 June 2004; accepted 9 July 2004)

\begin{abstract}
The presence of cochlear-based compression at low frequencies was investigated by measuring phase effects in harmonic maskers. In normal-hearing listeners, the amount of masking produced depends strongly on the phase relationships between the individual masker components. This effect is thought to be determined primarily by properties of the cochlea, including the phase dispersion and compressive input-output function of the basilar membrane. Thresholds for signals of 250 and $1000 \mathrm{~Hz}$ were measured in harmonic maskers with fundamental frequencies of 12.5 and $100 \mathrm{~Hz}$ as a function of the masker phase curvature. Results from 12 listeners with sensorineural hearing loss showed reduced masker phase effects, when compared with data from normal-hearing listeners, at both $250-$ and $1000-\mathrm{Hz}$ signal frequencies. The effects of hearing impairment on phase-related masking differences were not well simulated in normal-hearing listeners by an additive white noise, suggesting that the effects of hearing impairment are not simply due to reduced sensation level. Maximum differences in masked threshold were correlated with auditory filter bandwidths at the respective frequencies, suggesting that both measures are affected by a common underlying mechanism, presumably related to cochlear outer hair cell function. The results also suggest that normal peripheral compression remains strong even at $250 \mathrm{~Hz}$. (c) 2004 Acoustical Society of America. [DOI: 10.1121/1.1786852]
\end{abstract}

PACS numbers: 43.66.Ba, 43.66.Dc, 43.66.Sr [GK]

Pages: $2248-2257$

\section{INTRODUCTION}

Changing the phase relationships of components within a harmonic tone complex masker can have dramatic effects on the ability to detect a signal embedded in the masker. In general, waveforms that produce highly modulated patterns of temporal activity in the auditory periphery lead to lower thresholds than waveforms that produce a relatively unmodulated temporal envelope. Differences in masked threshold between such temporally "peaked" or "flat" maskers can in some cases exceed $25 \mathrm{~dB}$. These masking effects can be explained in two (not mutually exclusive) ways. First, listeners may be able to make use of the momentary high signalto-masker ratios in low-level epochs of the modulated masker envelopes to improve signal detection by "listening in the valleys" (Buus, 1985; Kohlrausch and Sander, 1995). Second, fast-acting compression of the waveform in the auditory periphery reduces the effective rms level of a modulated masker, relative to that of an unmodulated masker (Carlyon and Datta, 1997a; Oxenham and Dau, 2001a). The second explanation, based on peripheral compression, is consistent with the finding that hearing-impaired listeners generally show little or no effect of masker phase on signal threshold (Summers and Leek, 1998; Summers, 2000). A

${ }^{\text {a)} E l e c t r o n i c ~ m a i l: ~ o x e n h a m @ m i t . e d u ~}$ common cause of hearing impairment is a loss of outer hair cell function, which in turn leads to a reduction or loss of the basilar membrane's compressive input-output function (e.g., Ruggero and Rich, 1991; Oxenham and Plack, 1997). Thus, masker phase effects may provide an indirect measure of basilar-membrane compression.

One apparent anomaly in the compression theory of masker phase effects is that large effects continue to be found for normal-hearing listeners down to signal frequencies as low as $125 \mathrm{~Hz}$ (Oxenham and Dau, 2001b). In contrast, earlier physiological and psychophysical studies had concluded that cochlear nonlinearity is reduced or even absent at low characteristic frequencies (CFs) (Cooper and Yates, 1994; Hicks and Bacon, 1999; Plack and Oxenham, 2000). At least three interpretations of this apparent discrepancy are possible.

The first interpretation relates to the masker fundamental frequencies $(F 0 \mathrm{~s})$ used at low signal frequencies. At a signal frequency of $125 \mathrm{~Hz}$, Oxenham and Dau (2001b) used a masker $F 0$ of $12.5 \mathrm{~Hz}$, which corresponds to a period of 80 ms. As shown in an earlier study (Oxenham and Dau, 2001a), if the period of the masker is large compared with the integration period used by the auditory system to detect the signal, compression ceases to be necessary to explain masker phase effects. According to this interpretation, there- 
fore, the continued masker phase effects at very low signal frequencies may be due simply to the very long masker periods, enabling listening in the valleys (explanation 1, above), rather than to peripheral compression.

The second interpretation relates to whether peripheral compression is indeed reduced at low CFs. The question has not yet been settled conclusively via direct measurements of basilar-membrane motion because of the technical difficulties associated with accessing the apex of the cochlea without causing structural damage (Rhode and Cooper, 1996). As pointed out in an earlier study (Plack and Oxenham, 2000), most psychophysical (Oxenham and Plack, 1997; Nelson et al., 2001) and indirect physiological (Cooper and Yates, 1994) estimates of basilar-membrane compression rely on the assumption that a given place along the basilar membrane responds linearly to tones well below its CF. While this assumption is supported by basilar-membrane measurements in the base of the cochlea, its extrapolation to apical mechanics may not be justified. In fact, recent psychophysical studies using estimates of compression that do not make the assumption of off-frequency linearity have concluded that compression remains strong down to at least $250 \mathrm{~Hz}$ (LopezPoveda et al., 2003; Plack and Drga, 2003; Plack and O'Hanlon, 2003a). Thus, according to this interpretation, the large masker phase effects at low signal frequencies do in fact reflect strong peripheral compression at low CFs, and earlier studies suggesting reduced compression at apex of the cochlea are in error.

The third interpretation is that the masker phase differences are due to compression, but that the compression is located more centrally than the basilar membrane, at least for low signal frequencies. Along these lines, Zeng and Shannon (1994), using loudness functions derived from cochlearimplant patients, proposed that a lack of peripheral compression at low CFs may be compensated for by stronger central compression. The psychophysical studies inferring strong compression at low frequencies in normal-hearing listeners (Lopez-Poveda et al., 2003; Plack and Drga, 2003; Plack and O'Hanlon, 2003) cannot rule out the possibility that the compression actually occurs at a later stage in the auditory pathway.

This study attempts to distinguish between these interpretations by comparing the results from normal-hearing listeners with those from listeners with sensorineural hearing impairment. The rationale is as follows: if peripheral compression is present at low frequencies, then listeners with cochlear hearing loss at low frequencies are likely to exhibit reduced peripheral compression, presumably due to outer hair cell loss or dysfunction (Oxenham and Plack, 1997; Moore et al., 1999). Thus, if peripheral compression can account for masker phase differences (interpretation 2), then the reduction in masker phase effects found for hearingimpaired listeners at high signal frequencies (Summers and Leek, 1998; Summers, 2000) should also be found at low signal frequencies. Conversely, if the masker phase effects found in normal-hearing listeners at low frequencies are not due to peripheral compression, but to the long masker periods resulting from very low fundamental frequencies (interpretation 1), or to a more central compression (interpretation
3) then a loss of peripheral compression due to hearing loss should not affect results, and so the data from normalhearing and hearing-impaired listeners should be similar.

The present study also tests the relationship between masker phase effects and auditory filter bandwidths, which have been found to correlate strongly with estimates of peripheral compression (Moore et al., 1999). If peripheral compression underlies masker phase effects at all signal frequencies, then a strong correlation should exist between auditory filter bandwidth and the size of the masker phase effects at all frequencies. On the other hand, if peripheral compression underlies masker phase effects only at high frequencies, then the correlation should exist only at high signal frequencies. Finally, if peripheral compression is not responsible for masker phase effects, we might expect little or no correlation between the two measures.

Data were collected at signal frequencies of 250 and $1000 \mathrm{~Hz}$ from 12 hearing-impaired listeners with mild and moderate losses at the test frequencies. Three normal-hearing listeners served as controls. Thresholds were measured at both signal frequencies for harmonic complex maskers with an $F 0$ of $12.5 \mathrm{~Hz}$ (to measure masker phase effects) and for notched-noise maskers (to measure auditory filter bandwidths). At the higher signal frequency $(1000 \mathrm{~Hz})$, thresholds were also measured with a higher masker $F 0$ of $100 \mathrm{~Hz}$ to allow a more direct comparison with previous studies using hearing-impaired listeners (Summers and Leek, 1998).

\section{EXPERIMENT 1: MASKER PHASE EFFECTS AT 250 AND $1000 \mathrm{~Hz}$}

\section{A. Methods}

\section{Stimuli}

Thresholds were measured for a sinusoidal signal in the presence of a simultaneous masker. Signal frequencies were 250 and $1000 \mathrm{~Hz}$. The total signal duration was $260 \mathrm{~ms}$, gated with 30-ms raised-cosine ramps and temporally centered in a $320-\mathrm{ms}$ (total duration) masker, which was gated with $10-\mathrm{ms}$ raised-cosine ramps. The signal was added to the masker with a starting phase that was selected randomly from trial to trial. The masker was a harmonic tone complex with components between $0.4 f_{s}$ and $1.6 f_{s}$, where $f_{s}$ is the signal frequency. The phases of the components were selected according to a modification of Schroeder's (1970) equation, as used previously by Lentz and Leek (2001) and Oxenham and Dau (2001b)

$$
\theta_{n}=C \pi n(n-1) / N, \quad-1 \leqslant C \leqslant 1 .
$$

A Schroeder positive $\left(m_{+}\right)$or Schroeder negative $\left(m_{-}\right)$ complex is generated when $C=1$ or $C=-1$, respectively. Schroeder complexes are notable for their very flat temporal envelopes and can be characterized as either a rising $\left(m_{-}\right)$or falling $\left(m_{+}\right)$linear frequency glide, which repeats at a rate corresponding to the $F 0$. When $C=0$, a sine-phase complex is generated. The phase curvature of the complex is

$$
\frac{d^{2} \theta}{d f^{2}}=C \frac{2 \pi}{N F 0^{2}} .
$$


TABLE I. Ages and audiometric thresholds of the hearing-impaired listeners.

\begin{tabular}{|c|c|c|c|c|c|c|c|c|}
\hline \multirow[b]{2}{*}{ Subject } & \multirow[b]{2}{*}{ Age } & \multirow[b]{2}{*}{ Ear tested } & \multicolumn{6}{|c|}{ Audiometric threshold in test ear (dB HL) } \\
\hline & & & 250 & 500 & 1000 & 2000 & 4000 & 8000 \\
\hline HI1 & 83 & $\mathrm{R}$ & 45 & 55 & 70 & 60 & 60 & 80 \\
\hline HI 2 & 37 & $\mathrm{~L}$ & 50 & 45 & 45 & 45 & 40 & 45 \\
\hline HI3 & 80 & $\mathrm{~L}$ & 55 & 60 & 60 & 45 & 45 & 30 \\
\hline HI 4 & 34 & $\mathrm{R}$ & 30 & 30 & 35 & 45 & 50 & 55 \\
\hline HI5 & 36 & $\mathrm{R}$ & 50 & 50 & 55 & 55 & 55 & 65 \\
\hline HI6 & 60 & $\mathrm{R}$ & 30 & 40 & 45 & 40 & 45 & 60 \\
\hline HI7 & 47 & $\mathrm{~L}$ & 35 & 40 & 50 & 60 & 50 & 75 \\
\hline HI8 & 51 & $\mathrm{~L}$ & 50 & 50 & 45 & 55 & 45 & 35 \\
\hline HI9 & 33 & $\mathrm{R}$ & 40 & 50 & 45 & 60 & 85 & $>110$ \\
\hline HI10 & 36 & $\mathrm{R}$ & 55 & 55 & 60 & 60 & 65 & 70 \\
\hline HI11 & 53 & $\mathrm{~L}$ & 50 & 45 & 50 & 50 & 55 & 65 \\
\hline HI12 & 50 & $\mathrm{R}$ & 55 & 65 & 55 & 70 & 60 & 55 \\
\hline
\end{tabular}

By varying the $C$ value from -1 to 1 , a range of masker phase curvatures, or frequency sweep rates, can be generated. This leads to systematic changes in signal threshold, which are thought to reflect the interactions between the masker phase curvature and that of the basilar membrane, which in turn produce varying degrees of envelope modulation in the auditory response to the masker (Oxenham and Dau, 2001b). The overall level of the masker was set to 93 $\mathrm{dB}$ SPL. This relatively high level was selected in an attempt to ensure that all masked thresholds would lie well above the absolute thresholds of the hearing-impaired listeners.

The stimuli were generated digitally at a sampling rate of $48 \mathrm{kHz}$ and converted to analog signals by a 24-bit DAC including reconstruction filtering (SEKD ADSP 2496). The stimuli were presented to the listener via Sennheiser HD 580 headphones. Listeners were seated in a double-walled soundattenuating booth. Sounds were presented monaurally. For the hearing-impaired listeners the ear with the lower audiometric thresholds at the two test frequencies was chosen for testing.

\section{Procedure}

An adaptive three-interval three-alternative forcedchoice procedure was used in conjunction with a 2-down, 1-up tracking rule to estimate the $70.7 \%$-correct point on the psychometric function (Levitt, 1971). Each interval in a trial was separated by an interstimulus interval (ISI) of $500 \mathrm{~ms}$. The intervals were marked on a computer monitor and feedback was provided after each trial. Listeners responded via the computer keyboard or mouse. The initial step size was 5 $\mathrm{dB}$, which was reduced to $2 \mathrm{~dB}$ after the first four reversals. Threshold was defined as the mean of the remaining six reversals. Reported thresholds are the mean of four runs. Absolute thresholds for the two signal frequencies were also collected for each subject, using the same adaptive procedure as for the masked thresholds, with the exception that each threshold reported is the mean of three, rather than four, runs. The conditions were presented in random order, with all conditions being presented before embarking on the next repetition. The order of presentation of the conditions was selected randomly for each listener and each repetition. Measure- ments were made in 2-h sessions, including many short breaks. No more than one session per listener was completed in any one day.

\section{Listeners}

Twelve hearing-impaired and three normal-hearing listeners took part in the study. The second author served as one of the normal-hearing listeners (NH3). All the hearingimpaired listeners were paid for their participation, while the other two normal-hearing listeners were students who participated on a voluntary basis. The hearing-impaired listeners all had bilateral hearing loss. All losses were diagnosed as being of cochlear origin, as indicated by lack of an air-bone gap and evidence of loudness recruitment. Audiometric thresholds for the hearing-impaired listeners, along with their ages, are shown in Table I. The normal-hearing listeners were aged between 25 and 36 years and had audiometric thresholds of no more than $10 \mathrm{~dB}$ HL at the test frequencies. All the listeners (both hearing impaired and normal hearing) had some previous experience in psychoacoustic tests, including masking experiments. They were given practice until their performance appeared to be stable. This generally took about $2 \mathrm{~h}$.

\section{B. Results}

The results are shown in Fig. 1 for the individual subjects. Data from the three normal-hearing listeners are shown in the bottom row; the remaining data are from the 12 hearing-impaired listeners. Circles represent thresholds with a $250-\mathrm{Hz}$ signal; squares represent thresholds with a $1000-\mathrm{Hz}$ signal. Filled and open symbols denote thresholds using a $12.5-\mathrm{Hz}$ and $100-\mathrm{Hz}$ masker $F 0$, respectively. Absolute thresholds are also shown for the hearing-impaired listeners and are shaded gray. Absolute thresholds for the normalhearing listeners were all below $20 \mathrm{~dB}$ SPL (mean thresholds: $12.1 \mathrm{~dB}$ SPL at $250 \mathrm{~Hz}$ and $6.4 \mathrm{~dB}$ SPL at $1000 \mathrm{~Hz}$ ). With the exception of $\mathrm{HI} 12$ at $1000 \mathrm{~Hz}$, masked thresholds were always at least $6 \mathrm{~dB}$ (and in most cases more than 10 dB) above absolute threshold.

In the normal-hearing listeners, the pattern of results is similar to that found in previous studies (e.g., Oxenham and Dau, 2001b): thresholds vary considerably with masker 


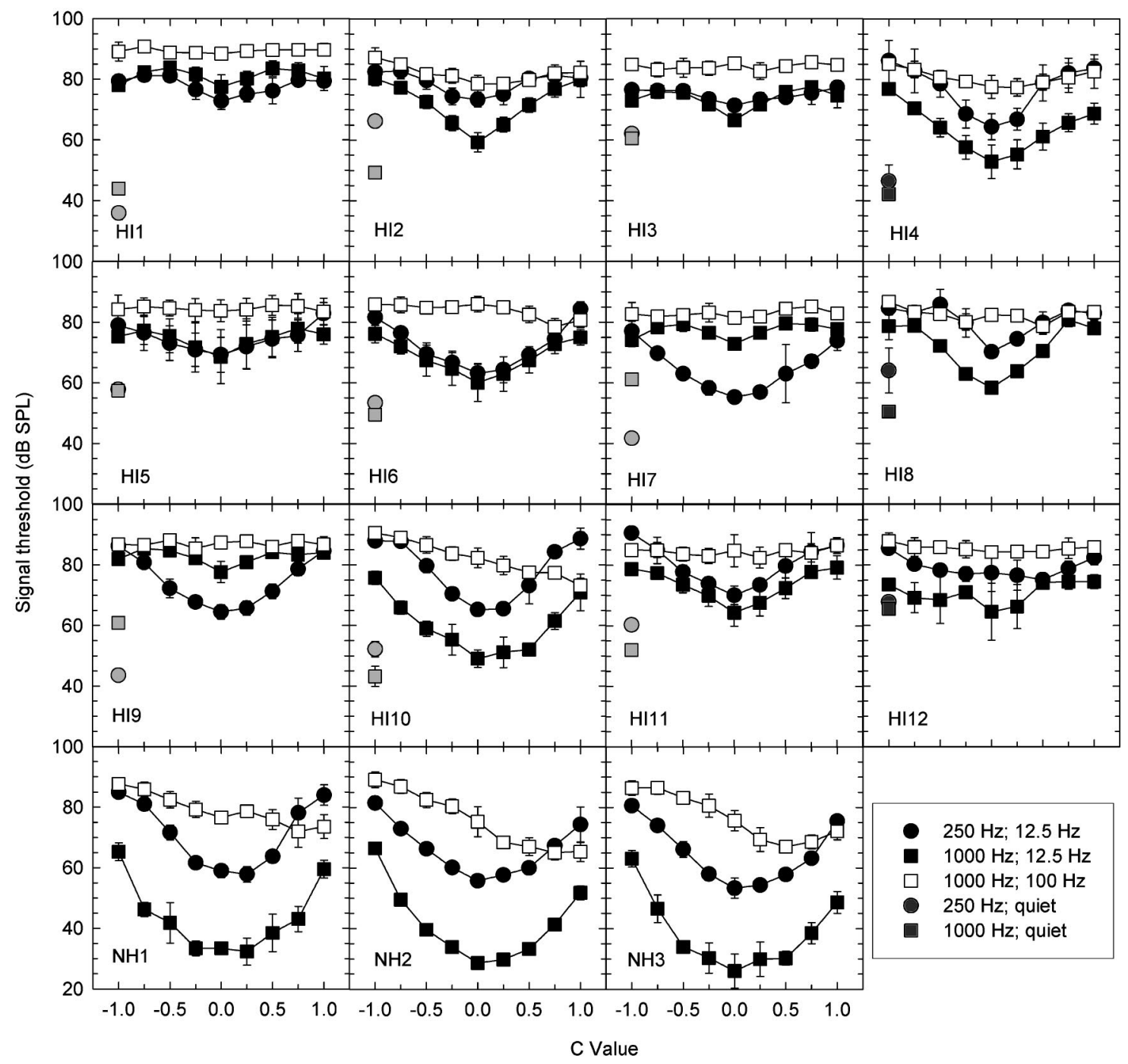

FIG. 1. Individual masked thresholds in the harmonic tone complex maskers, as a function of masker phase curvature, denoted by $C$. Different symbols represent different conditions, as shown in the legend. Error bars represent \pm 1 s.d. of the mean.

phase curvature and reach a minimum at a $C$ value that depends on the interaction between the masker phase curvature and the phase curvature of the auditory periphery. In good agreement with the current data, phase curvature estimates from our previous study (Oxenham and Dau, 2001b) predict that the $C$ value at the minimum for the $1000-\mathrm{Hz}$ signal should be around 1 for the $100-\mathrm{Hz} F 0$ and between 0 and 0.25 for the $12.5-\mathrm{Hz} F 0$. The minimum around $0-0.25$ found with a signal frequency of $250 \mathrm{~Hz}$ is also in good agreement with the results of our earlier study. The larger difference between maximum and minimum threshold found with decreasing $F 0$ at a given signal frequency has been reported in earlier studies (Kohlrausch and Sander, 1995; Oxenham and Dau, 2001b).

Results from the hearing-impaired listeners show a somewhat different pattern of results. Where minima in the masking functions are visible, they tend to occur at the same $C$ values as for normal-hearing listeners. In general, however, the effect of masker phase curvature on thresholds is much reduced in the hearing-impaired listeners at both signal frequencies. The effects of masker phase relationships were quantified for each listener by subtracting the minimum from the maximum masked threshold in a given condition. These differences are shown in Table II. It can be seen that most hearing-impaired listeners exhibit reduced masker phase effects in all three conditions. These data and their implications for the possible underlying mechanisms are considered further below (Sec. IV).

\section{Effects of background noise in normal hearing}

In general, hearing loss influences masker phase effects at both 250 and $1000 \mathrm{~Hz}$. Following from the reasoning laid out in the Introduction, this suggests that cochlear compression may remain relatively strong in normal hearing, even at frequencies as low as $250 \mathrm{~Hz}$, and that this compression may be reduced in listeners with hearing impairment. However, before proceeding under this assumption, it is important to test for the possibility that the effects can be accounted for simply by the elevated absolute thresholds in the hearingimpaired listeners. Studies attempting to simulate hearing impairment by using a background noise to elevate thresholds have come to various conclusions. Although most researchers agree that cochlear hearing impairment cannot be 
TABLE II. Difference in $\mathrm{dB}$ between the maximum and minimum thresholds in each condition of experiment 1 . This value provides an indication of the overall masker phase effect in individual listeners.

\begin{tabular}{llll}
\hline \hline & \multicolumn{3}{c}{ Condition } \\
\cline { 2 - 4 } Listener & $250 \mathrm{~Hz} ; 12.5 \mathrm{~Hz}$ & $1000 \mathrm{~Hz} ; 12.5 \mathrm{~Hz}$ & $1000 \mathrm{~Hz} ; 100 \mathrm{~Hz}$ \\
\hline HI1 & 8.5 & 6.3 & 2.4 \\
HI2 & 9.4 & 21.0 & 8.7 \\
HI3 & 5.9 & 10.9 & 2.9 \\
HI4 & 21.8 & 24.0 & 7.8 \\
HI5 & 13.6 & 7.3 & 2.1 \\
HI6 & 21.1 & 16.1 & 7.5 \\
HI7 & 21.8 & 6.8 & 3.8 \\
HI8 & 15.6 & 22.4 & 8.1 \\
HI9 & 21.8 & 7.8 & 2.8 \\
HI10 & 23.4 & 26.7 & 17.4 \\
HI11 & 20.6 & 14.9 & 4.0 \\
HI12 & 10.3 & 9.9 & 3.7 \\
NH1 & 27.0 & 32.9 & 15.7 \\
NH2 & 25.7 & 37.8 & 24.1 \\
NH3 & 26.2 & 37.1 & 19.4 \\
\hline \hline
\end{tabular}

recreated in normal hearing simply by adding masking noise (Fabry and Van Tasell, 1986; Leek et al., 1987; Dubno and Schaefer, 1992; Leek and Summers, 1993), there have been some studies showing certain aspects of the impairment can be successfully simulated in that way (e.g., Humes et al., 1987; Zurek and Delhorne, 1987; Dubno and Schaefer, 1992). Also, masker phase effects in normal-hearing listeners are reduced at low overall levels (Carlyon and Datta, 1997b; Oxenham and Dau, 2001b). This has been assumed to be due to more linear cochlear processing at low levels, but might conceivably be due to some other cause associated with low sensation levels.

\section{Methods}

To test the effect of sensation level on masker phase effects, the three normal-hearing listeners from experiment 1 were tested again in an additional background of white Gaussian noise, designed to raise the threshold of the signal to levels comparable to the absolute thresholds of some of our hearing-impaired listeners. Signal thresholds were measured for all the harmonic tone complex conditions tested in the main part of experiment 1 . The stimuli and procedure were identical to those used there, with the exception that a Gaussian white noise, bandpass filtered between 100 and $6000 \mathrm{~Hz}$ with a spectrum level of $27 \mathrm{~dB}$ SPL in the passband, was gated on and off synchronously with the complex-tone masker. This raised detection thresholds of the signal in the noise alone to around $45 \mathrm{~dB}$ SPL at both signal frequencies for the three normal-hearing listeners.

\section{Results}

Individual results from the three normal-hearing listeners are shown in the three rows of Fig. 2. Columns 1-3 represent the $250-\mathrm{Hz}, 1000-\mathrm{Hz}(12.5-\mathrm{Hz} F 0)$, and $1000-\mathrm{Hz}$ (100-Hz F0), respectively. Corresponding data from experiment 1, without the background noise, are replotted in each panel as lines without symbols. Gray symbols show the signal thresholds in the noise alone for the 250-Hz (column 1) and $1000-\mathrm{Hz}$ (columns 2 and 3 ) signals. In the $250-\mathrm{Hz}$ signal condition (left column) and the $1000-\mathrm{Hz}$ signal condition with the $100-\mathrm{Hz}$ masker $F 0$ (right column), the additional noise masker has essentially no effect on masked thresholds. It is only in the $1000-\mathrm{Hz}$ signal condition with the $12.5-\mathrm{Hz}$ masker, where thresholds in the complex-tone masker drop below the signal threshold in noise alone, that the effect of

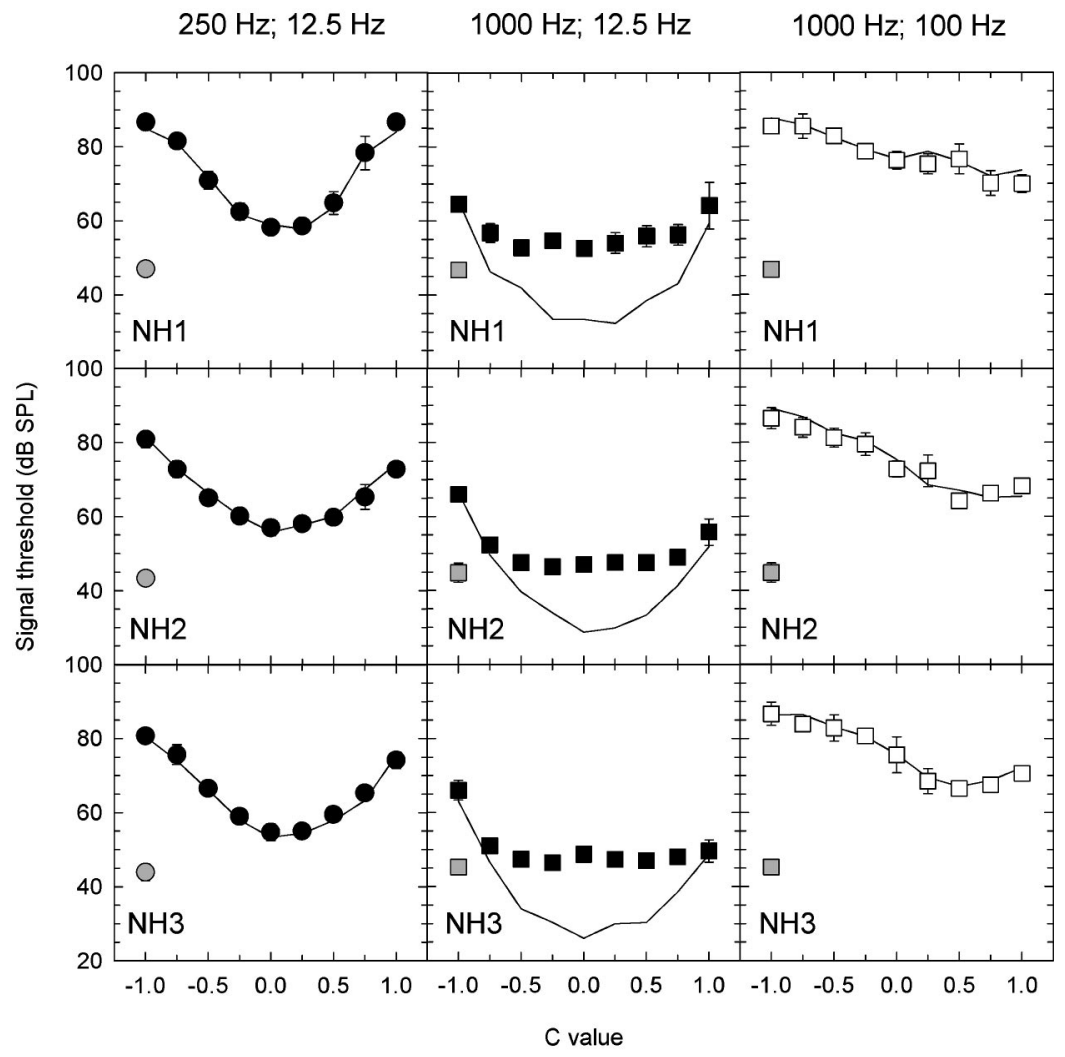

FIG. 2. Individual masked thresholds from three normal-hearing listeners in the harmonic tone complex maskers, combined with a Gaussian white noise. Each column presents data from a different condition, as shown in the column headers. Thresholds in the noise alone are shown as gray symbols; thresholds in the harmonic tone complex alone are replotted from Fig. 1 as lines. 
the noise masker becomes apparent. Even here, it is only when the signal threshold would otherwise be within about 5 $\mathrm{dB}$ of its masked threshold in the noise alone that thresholds are affected.

The pattern of results found in the normal-hearing listeners with an additional background noise does not resemble the general pattern of results of the hearing-impaired listeners (see Fig. 1). With the hearing-impaired listeners, masker phase effects were generally less than those found for normal-hearing listeners, even though masked thresholds for the hearing-impaired listeners were in most cases more than $10 \mathrm{~dB}$ above absolute threshold. Thus, it appears that the signal's reduced sensation level cannot account for the differences found between normal-hearing and hearingimpaired listeners. This is interesting as it provides an example of an auditory task in which additive noise does not provide an adequate simulation of moderate hearing loss in normal-hearing listeners. Other examples include temporal integration, or the decrease in signal threshold with increasing signal duration (e.g., Oxenham et al., 1997), and the ability to recognize speech in amplitude-modulated backgrounds (Eisenberg et al., 1995).

\section{EXPERIMENT 2: AUDITORY FILTER SHAPES AT 250 AND $1000 \mathrm{~Hz}$}

This experiment measured auditory filter bandwidths at 250 and $1000 \mathrm{~Hz}$ in the same 12 hearing-impaired listeners as used in experiment 1 . Only one normal-hearing listener (NH3) was tested. As mentioned in the Introduction, if a loss of peripheral compression in the hearing-impaired listeners can account for the reduced masker phase effects, then we should find a correlation between the difference in maximum and minimum thresholds found in experiment 1 and the auditory filter bandwidths, which are also thought to be highly correlated with peripheral compression (Moore et al., 1999).

\section{A. Method}

The signals and the temporal parameters of both the masker and signal were identical to those used in experiment 1. However, instead of the masker being a harmonic tone complex, it consisted of two spectral bands of Gaussian noise, each with a bandwidth of $0.4 f_{s}$, centered below and above the signal frequency. The two noise bands were either contiguous ( 0 notch condition) or were spaced apart in frequency, thereby introducing a spectral notch between them. The notches were defined in terms of the frequency separation $(\Delta f)$ between the signal frequency and the nearer edge of one of the noise bands, normalized by the signal frequency (i.e., $\Delta f / f_{s}$ ). The noise bands were placed either symmetrically or asymmetrically around the signal frequency. In the symmetric conditions, the notch values were $0,0.2$, and 0.4 . In the asymmetric conditions, the notch on one side of the signal was 0.2 , while on the other side it was 0.4 . This led to the same five conditions (three symmetric and two asymmetric) that were deemed by Stone et al. (1992) sufficient to produce reasonably accurate estimates of auditory filter shape. The spectrum level of the masker was
TABLE III. Individual equivalent rectangular bandwidths (ERBs) of auditory filters measured at 250 and $1000 \mathrm{~Hz}$, using a fixed spectrum-level noise of 60 and $50 \mathrm{~dB}$ SPL, respectively. The values are normalized to the signal frequency (i.e., $\mathrm{ERB} / f_{\mathrm{s}}$ ). The values in brackets are the rms errors (in $\mathrm{dB}$ ) of the signal threshold predictions using the fitted filter function, which was a symmetric roex $(p)$ filter. Missing values $(\cdots)$ indicate conditions in which more than one data point was within $5 \mathrm{~dB}$ of absolute threshold.

\begin{tabular}{lcc}
\hline \hline & \multicolumn{2}{c}{ Signal frequency } \\
\cline { 2 - 3 } Listener & $250 \mathrm{~Hz}$ & $1000 \mathrm{~Hz}$ \\
\hline HI1 & $0.43(0.47)$ & $1.23(1.20)$ \\
HI2 & $0.92(0.55)$ & $0.38(1.47)$ \\
HI3 & $0.52(1.27)$ & $\ldots$ \\
HI4 & $0.37(0.77)$ & $0.27(2.09)$ \\
HI5 & $0.41(0.89)$ & $0.36(1.18)$ \\
HI6 & $0.38(1.10)$ & $0.32(2.13)$ \\
HI7 & $0.35(0.55)$ & $2.00(0.68)$ \\
HI8 & $0.44(0.71)$ & $0.35(1.32)$ \\
HI9 & $0.43(0.49)$ & $2.00(0.45)$ \\
HI10 & $0.29(0.60)$ & $0.16(1.39)$ \\
HI11 & $0.79(1.16)$ & $0.57(1.10)$ \\
HI12 & $\cdots$ & $\cdots$ \\
NH3 & $0.39(0.84)$ & $0.24(1.71)$ \\
\hline \hline
\end{tabular}

$60 \mathrm{~dB}$ SPL at $250 \mathrm{~Hz}$ and $50 \mathrm{~dB}$ SPL at $1000 \mathrm{~Hz}$, producing an overall level of around $83 \mathrm{~dB}$ SPL and $80 \mathrm{~dB}$ SPL at 250 and $1000 \mathrm{~Hz}$, respectively.

The method of testing was also identical to that used in experiment 1 , and again, four repetitions of each condition were averaged to compute the individual threshold estimates. Two exceptions occurred (both with subject HI10), where one run resulted in a threshold that exceeded the mean of the other three runs by more than 4 standard deviations. These two outliers were discarded and the mean of the remaining three runs in each of the two conditions was recorded.

\section{B. Results}

As expected from the extensive literature on the topic (e.g., Patterson et al., 1982; Tyler et al., 1984; Moore and Glasberg, 1986; Dubno and Schaefer, 1995; Moore et al., 1999; Baker and Rosen, 2002), changes in the masked signal thresholds of the hearing-impaired listeners as a function of masker notch width were less than those found for normalhearing listeners. Masked thresholds were on the whole well above the absolute thresholds of the individual listeners. On average, the lowest thresholds in the notched-noise conditions exceeded absolute thresholds by more than $10 \mathrm{~dB}$. However, in 4 of the 12 hearing-impaired listeners, at least one masked threshold fell within $5 \mathrm{~dB}$ of absolute threshold. For the purposes of analysis, these thresholds were excluded from the fitting procedure. If more than one of the five thresholds within a condition was excluded, a fit was not attempted. This procedure resulted in the exclusion of the $1000-\mathrm{Hz}$ data of listener $\mathrm{HI} 3$ and of both the $250-\mathrm{Hz}$ and $1000-\mathrm{Hz}$ data of listener HI12.

Frequency selectivity was quantified in the individual listeners by deriving auditory filter shapes from the data, using the rounded-exponential, $\operatorname{roex}(p)$, model (e.g., Patterson et al., 1982). Initially, we allowed the parameter $p$, which defines the slope of the filter function, to vary inde- 


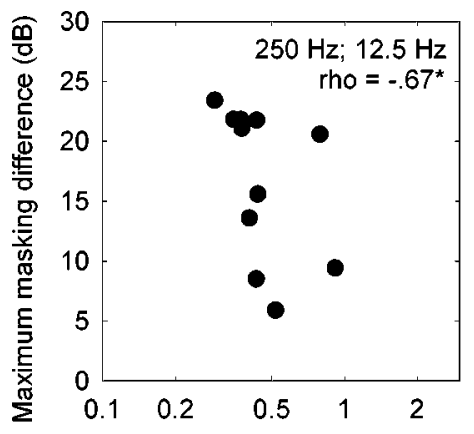

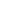

(1)

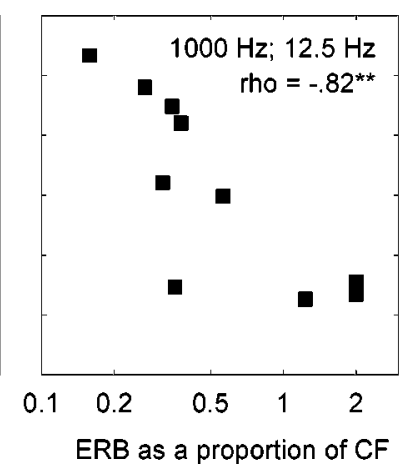

ERB as a proportion of CF

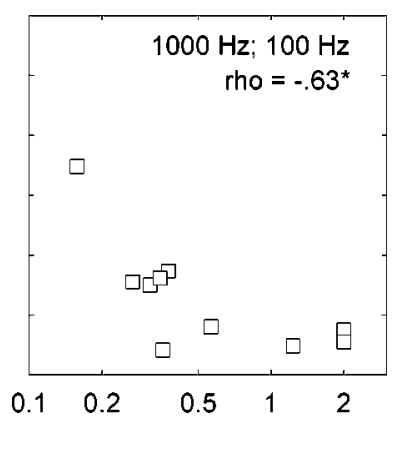

FIG. 3. Maximum masking difference in harmonic tone complex maskers, plotted as a function of ERB. Each data point represents one hearingimpaired listener. The frequencies shown are the signal frequency and masker $F 0$, respectively. The Spearman rank correlation coefficient is also shown in each panel. One and two asterisks denote statistical significance at the $p=0.05$ and $p=0.01$ levels, respectively (one-tailed tests). pendently on the lower and upper side of the filter. However, for some of the hearing-impaired listeners, where the slope of the masking function was very shallow, this led to very poorly defined filter estimates, probably because of the small number of data points (5) used to fit the model. We then restricted the model to a single free parameter, $p$, which defined the slope on both sides of the filter. By using only one parameter, we assume the filter is symmetric. Note, however, that this does not imply that the model cannot predict threshold differences in the asymmetric notch conditions. This is because the model "detects" the signal in the filter with the lowest signal-to-noise ratio, which does not necessarily have a CF corresponding to the signal frequency ("offfrequency listening"). Furthermore, this model simplification led to only a small increase in prediction errors (mean increase in rms error $<0.2 \mathrm{~dB}$ at both 250 and $1000 \mathrm{~Hz}$ ).

The results from the fitting procedure were used to derive equivalent rectangular bandwidths (ERBs) for each listener at each of the two signal frequencies. These values are given in Table III, normalized by the signal frequency. The ERBs were calculated by integrating the area under the derived filter from 0 to $2 f_{s} \mathrm{~Hz}$. Thus, the maximum possible normalized ERB value was 2, which essentially implies no measurable frequency selectivity. This was the case for 2 of the 12 subjects (HI7 and HI9) at a signal frequency of 1000 $\mathrm{Hz}$. For these two subjects, thresholds for a total notch width of $800 \mathrm{~Hz}$ were within 1 or $2 \mathrm{~dB}$ of those found with no notch. The ERBs from the normal-hearing listener are larger than the normative values provided by Glasberg and Moore (1990) of 0.21 and 0.13 at 250 and $1000 \mathrm{~Hz}$, respectively. This is at least in part due to the high overall level used in the current experiments, which is known to increase estimated filter bandwidths.

\section{ANALYSIS}

Experiment 1 found that masker phase effects were greatly reduced in hearing-impaired listeners at both 250 and $1000 \mathrm{~Hz}$. A previous study showed a similar lack of phase effects in hearing-impaired listeners (Summers and Leek, 1998). However, in that study, only two masker phase curvatures were tested $(C=1$ or -1$)$, leaving open the possibility that the smaller differences may have reflected only a shift along the $C$ axis in the maximum and minimum of the masking functions, rather than a reduction in the size of the effect itself. Such a shift might result from a change in the phase response of the impaired cochlea. For instance, for the
$1000-\mathrm{Hz}$ signal in the $100-\mathrm{Hz} F 0$ masker, the minimum of the masking function might shift to $C=0$ if the phase curvature of the impaired cochlea tended toward zero. Although there might be a hint of such a shift in 2 of the 12 listeners (HI2 and HI4), two other listeners (HI6 and HI10) show more normal minima, while the other 8 listeners show no reliable minima. Thus, the reduced phase effects in the hearing-impaired listeners do not seem to be due to a simple change in the phase curvature of the impaired cochlea. It is possible that a more complex change in the phase response, resulting in markedly nonuniform phase curvature in the impaired cochlea, could lead to none of the masker waveforms (which all have constant phase curvature) achieving maximal "peakiness." In that case, at least part of the reduction in phase effects could be due to complex changes in the phase response, rather than to changes in compression. However, current data suggest that the phase curvature of the normal cochlea is rather invariant over a large range of levels (Oxenham and Dau, 2001b; Shera, 2001), suggesting that perhaps it may remain relatively invariant after cochlear damage.

If the size of the masker phase effect instead provides an indirect estimate of cochlear nonlinearity or compression, then one might expect a strong correlation between it and auditory filter bandwidth estimates in the same subjects. This is because both measures are thought to reflect the functioning of the cochlea's outer hair cells. To test this, the relation between the masker phase effect, quantified using the maximum-minimum difference in threshold shown in Table II, and the ERBs given in Table III, was analyzed. Both measures were also compared with individual absolute thresholds. These relationships for the hearing-impaired listeners are shown in Figs. 3, 4, and 5. The numbers in the panels show the stimulus parameters and the Spearman rank correlation coefficients.

In Fig. 3, the maximum-minimum masking difference is plotted as a function of the ERB values, on a log scale. Correlations in all three conditions are significant, although the relationships are not very strong, with all three conditions exhibiting considerable scatter. Similarly weak but significant correlations were found between absolute threshold and ERB (Fig. 4), as has been found previously (e.g., Moore et al., 1999). The relationship between absolute threshold and the maximum-minimum masking difference (Fig. 5) appeared to be weaker, with only two of the three conditions exhibiting a statistically significant relationship. 


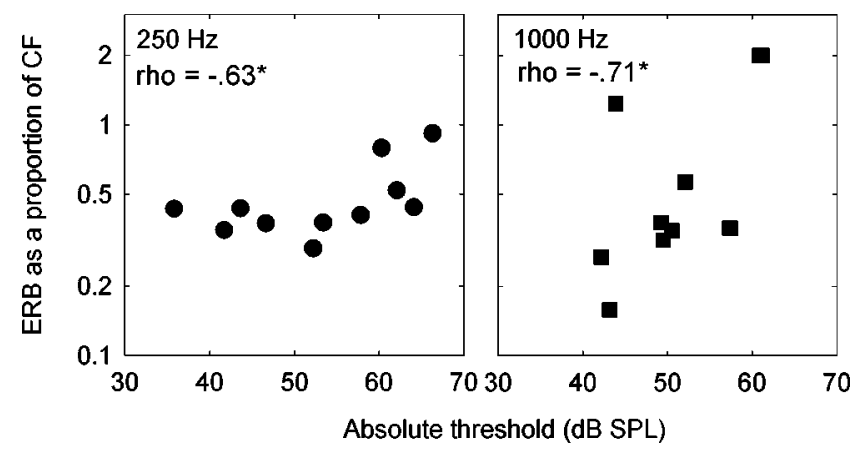

FIG. 4. Individual ERBs, plotted as a function of absolute threshold. Stimulus parameters and correlation coefficients are shown, as in Fig. 3.

Other potential correlations were also examined as controls. No measure at $250 \mathrm{~Hz}$ (absolute thresholds, maximum-minimum threshold difference, or ERB) was significantly correlated with any measure at $1 \mathrm{kHz}$. There were also no strong relationships between listener age and psychoacoustic measure. The correlation coefficient between listener age and maximum-minimum masking difference at $250 \mathrm{~Hz}$ did reach statistical significance $(\mathrm{rho}=0.62 ; p$ $=0.015$, one-tailed test; uncorrected for multiple comparisons), but the six other potential correlations with age (ERB at 250 and $1000 \mathrm{~Hz}$, maximum masking difference at 1000 $\mathrm{Hz}$ for 12.5 and $100 \mathrm{~Hz} F 0$, absolute threshold at 250 and $1000 \mathrm{~Hz})$ were all below rho $=0.3(p>0.2)$.

\section{DISCUSSION}

The results of experiment 1 suggest that the large masker phase effects observed at very low signal frequencies in our earlier study (Oxenham and Dau, 2001b) were not simply due to the low $F 0$ used. Instead, peripheral compression is likely to play a role. This conclusion is based on the fact that hearing-impaired listeners exhibited similarly reduced masker phase effects with an $F 0$ of $12.5 \mathrm{~Hz}$ at both signal frequencies of 250 and $1000 \mathrm{~Hz}$.

Our conclusion is in line with other recent studies, presenting evidence for strong compression in normal hearing at very low frequencies (Lopez-Poveda et al., 2003; Plack and Drga, 2003; Plack and O'Hanlon, 2003). The results of the present study extend these findings by showing that compression at low frequencies seems to be affected by cochlear hearing loss in the same way as compression at high frequencies. This makes retrocochlear compression (e.g., Zeng and
Shannon, 1994) a less likely explanation for the data from normal-hearing listeners in this and other studies of lowfrequency compression.

According to our hypothesis, frequency selectivity and masker phase sensitivity are both largely determined by the functioning of the cochlea's outer hair cells. In contrast, absolute threshold may reflect a number of different factors, including outer hair cell and inner hair cell loss or dysfunction, as well as possible retrocochlear components that are not easily distinguished from cochlear hearing loss. Thus, we might have expected ERB values and the maximum masking difference to be more strongly correlated with each other than with absolute threshold (see also Moore et al., 1999). This was not the case: essentially all three measures were weakly, but mostly significantly, correlated with each other. One explanation is that the measure of maximum masking difference does not reflect solely the functional status of the cochlear outer hair cells, as is likely to be the case for the ERB values. For instance, detection of a tone in a fluctuating periodic masker may involve some degree of temporal resolution. Although temporal resolution is generally not thought to be strongly affected by cochlear hearing loss, there exist certain patients, currently diagnosed with "auditory neuropathy" (Sininger and Starr, 2001), who exhibit extremely poor temporal resolution, as measured using amplitude modulation detection and gap detection (Zeng et al., 1999, 2001). It is at least conceivable that some more subtle temporal deficits, which are not apparent in standard tests for cochlear hearing loss, may affect thresholds in our masker phase thresholds, but not ERBs.

Another possible explanation for the relatively weak correlations is that the relationship between masker phase effects and the degree of outer hair cell loss is complicated by the contribution of filter bandwidth. Increasing the effective filter bandwidth leads to more masker components interacting and hence to greater maximum peakiness of the temporal envelope, which in turn leads to larger potential masking differences. Thus, outer hair cell damage or dysfunction results in a loss of peripheral compression, which may decrease masker phase effects, but also results in a widening of filter bandwidths, which may increase masker phase effects. It is conceivable that these two competing effects may interact differently in different listeners, resulting in weaker-than-expected correlations.

In summary, the maximum masking difference does not provide an easily interpretable quantitative estimate of co-

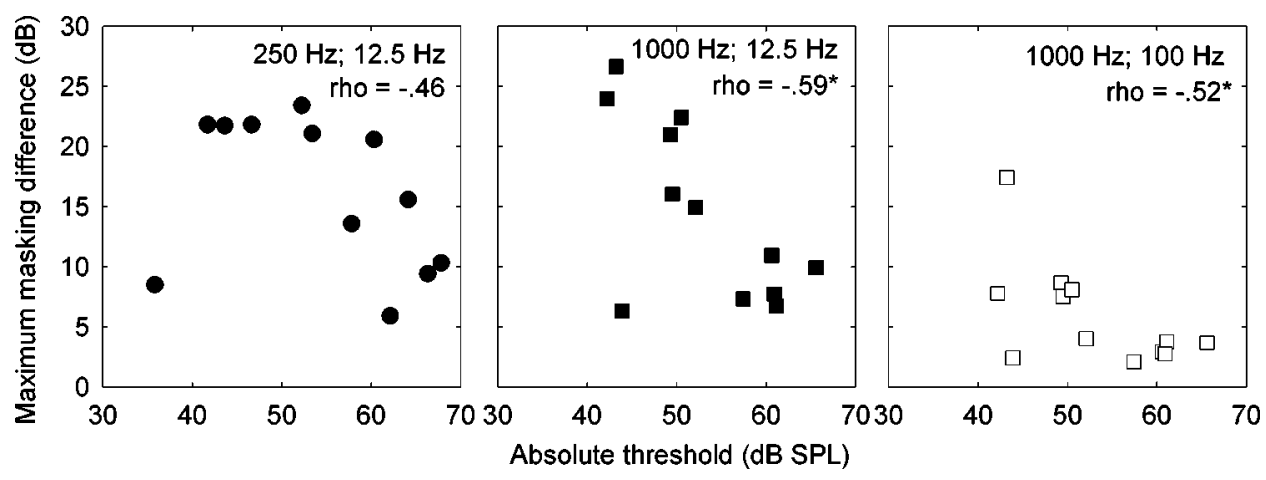

FIG. 5. Individual maximum masking differences, plotted as a function of absolute threshold. Stimulus parameters and correlation coefficients are shown, as in Fig. 3. 
chlear compression; measures such as masking additivity (Plack and O'Hanlon, 2003), and temporal masking curves (Lopez-Poveda et al., 2003; Plack and Drga, 2003) are probably more suitable. Nevertheless, while masker phase masking differences are not easily quantified in terms of amount of cochlear compression, the results from our hearingimpaired and normal-hearing listeners do provide reasonably strong qualitative support for continued peripheral compression at low $\mathrm{CFs}$ in normal hearing.

\section{SUMMARY AND CONCLUSIONS}

The results and analysis from experiments 1 and 2 can be summarized as follows:

(1) At both signal frequencies tested $(250$ and $1000 \mathrm{~Hz}$ ), effects of masker phase on signal thresholds were substantially less in hearing-impaired listeners than in normal-hearing listeners. This suggests that the masker phase differences found in normal hearing at low signal frequencies may be due to continued strong compression in apical regions of the normal cochlea, which is reduced or no longer present in the impaired cochlea.

(2) Additive white noise did not materially affect masker phase differences in normal-hearing listeners, unless the signal threshold was within $5 \mathrm{~dB}$ of its threshold in the noise alone. This suggests that the reduction in masker phase effects with hearing impairment is not due simply to the lower sensation level at which the stimuli were presented to the hearing-impaired listeners. The results provide a further example of a situation where moderate hearing loss is not adequately simulated by presenting masking noise to normal-hearing listeners.

(3) Correlations between the auditory filter bandwidth (ERB) and the maximum masking differences in the hearing-impaired listeners were statistically significant, but not overwhelming, suggesting that thresholds in the two tasks are mediated by overlapping, but not necessarily identical, mechanisms.

\section{ACKNOWLEDGMENTS}

This work was supported by the National Institutes of Health (NIDCD Grant R01 DC 03909 to A.J.O.) and the Deutsche Forschungsgemeinschaft (T.D.). The experiments were carried out at the laboratory of the work group Medizinische Physik at Oldenburg University while the first author was a fellow at the Hanse Institute for Advanced Study, Delmenhorst, Germany. We thank Müge Kaya from the Hörzentrum Oldenburg for assistance in recruiting and collecting data from the hearing-impaired listeners. Comments by Gerald Kidd and the reviewers helped improve the paper.

Baker, R. J., and Rosen, S. (2002). "Auditory filter nonlinearity in mild/ moderate hearing impairment," J. Acoust. Soc. Am. 111, 1330-1339.

Buus, S. (1985). "Release from masking caused by envelope fluctuations," J. Acoust. Soc. Am. 78, 1958-1965.

Carlyon, R. P., and Datta, A. J. (1997a). "Excitation produced by Schroederphase complexes: Evidence for fast-acting compression in the auditory system,” J. Acoust. Soc. Am. 101, 3636-3647.
Carlyon, R. P., and Datta, A. J. (1997b). "Masking period patterns of Schroeder-phase complexes: Effects of level, number of components, and phase of flanking components," J. Acoust. Soc. Am. 101, 3648-3657.

Cooper, N. P., and Yates, G. K. (1994). "Nonlinear input-output functions derived from the responses of guinea-pig cochlear nerve fibers: Variations with characteristic frequency," Hear. Res. 78, 221-234.

Dubno, J. R., and Schaefer, A. B. (1992). "Comparison of frequency selectivity and consonant recognition among hearing-impaired and masked normal-hearing listeners," J. Acoust. Soc. Am. 91, 2110-2121.

Dubno, J. R., and Schaefer, A. B. (1995). "Frequency selectivity and consonant recognition for hearing-impaired and normal-hearing listeners with equivalent masked thresholds," J. Acoust. Soc. Am. 97, 1165-1174.

Eisenberg, L. S., Dirks, D. D., and Bell, T. S. (1995). "Speech recognition in amplitude-modulated noise of listeners with normal and listeners with impaired hearing," J. Speech Hear. Res. 38, 222-233.

Fabry, D. A., and Van Tasell, D. J. (1986). "Masked and filtered simulation of hearing loss: Effects on consonant recognition," J. Speech Hear. Res. 29, 170-178.

Glasberg, B. R., and Moore, B. C. J. (1990). "Derivation of auditory filter shapes from notched-noise data," Hear. Res. 47, 103-138.

Hicks, M. L., and Bacon, S. P. (1999). "Psychophysical measures of auditory nonlinearities as a function of frequency in individuals with normal hearing," J. Acoust. Soc. Am. 105, 326-338.

Humes, L. E., Dirks, D. D., and Kincaid, G. E. (1987). "Recognition of nonsense syllables by hearing-impaired listeners and by noise masked normal listeners," J. Acoust. Soc. Am. 81, 765-773.

Kohlrausch, A., and Sander, A. (1995). "Phase effects in masking related to dispersion in the inner ear. II. Masking period patterns of short targets," J. Acoust. Soc. Am. 97, 1817-1829.

Leek, M. R., and Summers, V. (1993). "Auditory filter shapes of normalhearing and hearing-impaired listeners in continuous broadband noise," J. Acoust. Soc. Am. 94, 3127-3137.

Leek, M. R., Dorman, M. F., and Summerfield, Q. (1987). "Minimum spectral contrast for vowel identification by normal-hearing and hearingimpaired listeners," J. Acoust. Soc. Am. 81, 148-154.

Lentz, J. J., and Leek, M. R. (2001). "Psychophysical estimates of cochlear phase response: Masking by harmonic complexes," J. Assoc. Res. Otolaryngol. 2, 408-422.

Levitt, H. (1971). "Transformed up-down methods in psychoacoustics," J. Acoust. Soc. Am. 49, 467-477.

Lopez-Poveda, E. A., Plack, C. J., and Meddis, R. (2003). "Cochlear nonlinearity between 500 and $8000 \mathrm{~Hz}$ in listeners with normal hearing," J. Acoust. Soc. Am. 113, 951-960.

Moore, B. C. J., and Glasberg, B. R. (1986). "Comparisons of frequency selectivity in simultaneous and forward masking for subjects with unilateral cochlear impairments," J. Acoust. Soc. Am. 80, 93-107.

Moore, B. C. J., Vickers, D. A., Plack, C. J., and Oxenham, A. J. (1999). "Inter-relationship between different psychoacoustic measures assumed to be related to the cochlear active mechanism," J. Acoust. Soc. Am. 106, 2761-2778.

Nelson, D. A., Schroder, A. C., and Wojtczak, M. (2001). "A new procedure for measuring peripheral compression in normal-hearing and hearingimpaired listeners," J. Acoust. Soc. Am. 110, 2045-2064.

Oxenham, A. J., and Dau, T. (2001a). "Reconciling frequency selectivity and phase effects in masking," J. Acoust. Soc. Am. 110, 1525-1538.

Oxenham, A. J., and Dau, T. (2001b). "Towards a measure of auditory-filter phase response," J. Acoust. Soc. Am. 110, 3169-3178.

Oxenham, A. J., and Plack, C. J. (1997). "A behavioral measure of basilarmembrane nonlinearity in listeners with normal and impaired hearing," J. Acoust. Soc. Am. 101, 3666-3675.

Oxenham, A. J., Moore, B. C. J., and Vickers, D. A. (1997). "Short-term temporal integration: Evidence for the influence of peripheral compression," J. Acoust. Soc. Am. 101, 3676-3687.

Patterson, R. D., Nimmo-Smith, I., Weber, D. L., and Milroy, R. (1982). "The deterioration of hearing with age: Frequency selectivity, the critical ratio, the audiogram, and speech threshold," J. Acoust. Soc. Am. 72, $1788-1803$.

Plack, C. J., and Drga, V. (2003). "Psychophysical evidence for auditory compression at low characteristic frequencies," J. Acoust. Soc. Am. 113, $1574-1586$.

Plack, C. J., and O'Hanlon, C. G. (2003). "Forward masking additivity and auditory compression at low and high frequencies," J. Assoc. Res. Otolaryngol. 4, 405-415.

Plack, C. J., and Oxenham, A. J. (2000). "Basilar-membrane nonlinearity 
estimated by pulsation threshold," J. Acoust. Soc. Am. 107, 501-507.

Rhode, W. S., and Cooper, N. P. (1996). "Nonlinear mechanics in the apical turn of the chinchilla cochlea in vivo," Aud. Neurosci. 3, 101-121.

Ruggero, M. A., and Rich, N. C. (1991). "Furosemide alters organ of Corti mechanics: Evidence for feedback of outer hair cells upon the basilar membrane," J. Neurosci. 11, 1057-1067.

Schroeder, M. R. (1970). "Synthesis of low peak-factor signals and binary sequences with low autocorrelation," IEEE Trans. Inf. Theory 16, 85-89.

Shera, C. A. (2001). "Intensity invariance of fine time structure in basilarmembrane click response: Implications for cochlear mechanics," J. Acoust. Soc. Am. 110, 332-348.

Sininger, Y., and Starr, A. (2001). Auditory Neuropathy. (Singular, San Diego).

Stone, M. A., Glasberg, B. R., and Moore, B. C. J. (1992). "Simplified measurement of impaired auditory filter shapes using the notched-noise method," Br. J. Audiol. 26, 329-334.

Summers, V. (2000). "Effects of hearing impairment and presentation level on masking period patterns for Schroeder-phase harmonic complexes," J. Acoust. Soc. Am. 108, 2307-2317.
Summers, V., and Leek, M. R. (1998). "Masking of tones and speech by Schroeder-phase harmonic complexes in normally hearing and hearingimpaired listeners," Hear. Res. 118, 139-150.

Tyler, R. S., Hall, J. W., Glasberg, B. R., Moore, B. C. J., and Patterson, R. D. (1984). "Auditory filter asymmetry in the hearing impaired," J. Acoust. Soc. Am. 76, 1363-1368.

Zeng, F.-G., and Shannon, R. V. (1994). "Loudness-coding mechanisms inferred from electric stimulation of the human auditory system," Science 264, 564-566.

Zeng, F. G., Oba, S., Garde, S., Sininger, Y., and Starr, A. (1999). "Temporal and speech processing deficits in auditory neuropathy," NeuroReport 10, 3429-3435.

Zeng, F. G., Oba, S., Garde, S., Sininger, Y., and Starr, A. (2001). "Psychoacoustics and speech perception in auditory neuropathy," in Auditory Neuropathy, edited by Y. Sininger and A. Starr (Singular, San Diego).

Zurek, P. M., and Delhorne, L. A., (1987). "Consonant reception in noise by listeners with mild and moderate sensorineural hearing impairment," J. Acoust. Soc. Am. 82, 1548-1559. 